\title{
Evaluación de la permeabilidad al vapor de agua de películas de proteína de lactosuero/quitosano y su efecto sobre la respiración en banano recubierto
}

\author{
Evaluation of the water vapor permeability \\ for whey protein/chitosan films and its effect \\ on respiration of coated banana
}

Arce Ortiz, Kadaffi León (1), Ortega Villalba, Karen Johana (1), Ochoa-Martinez, Claudia Isabel (1), Vélez Pasos, Carlos (1)

(1) Grupo de investigación GIPAB, Universidad del Valle, Cali, Colombia.

Contacto: claudia.ochoa@correounivalle.edu.co

ReCibido: 6/3/2016 - APROBADO: 21/7/2016

\begin{abstract}
Resumen
Los recubrimientos y películas elaborados lactosuero (WPI) y quitosano (CHI) son conocidos por ser antifúngicos y por ser barrera para la transferencia de vapor de agua, logrando un efecto en la conservación de frutas. El objetivo de este trabajo fue determinar el efecto de un recubrimiento de WPI y CHI sobre la respiración del banano durante el almacenamiento en condiciones ambientales $\left(25 \pm 3^{\circ} \mathrm{C}\right.$ y $\left.75 \pm 5 \% \mathrm{HR}\right)$ y su relación con la permeabilidad al vapor de agua (PVA) de las películas. Para la elaboración del recubrimiento se evaluaron distintas concentraciones de WPI y CHI. Se empleó CHI al 75\% de desacetilación (solución de 1,2\%, 1,5\% y 1,8\% p/v) y WPI al 96\% (solución de $3 \%$ y $4 \% \mathrm{p} / \mathrm{v}$ ). La tasa de respiración se determinó realizando mediciones durante 55 minutos para cada tratamiento empleando un respirómetro digital. El mejor recubrimiento fue CHI 1,5\%-WPI 3\% donde la maduración y respiración fue controlada y el pico climatérico ocurrió el día 9 de almacenamiento con 109,84 mg CO $2 / \mathrm{kg}-\mathrm{h}$ (en la muestra control fue el día 7 con $172,71 \mathrm{mg} \mathrm{CO} / \mathrm{kg}-\mathrm{h}$ ). La permeabilidad al vapor de agua de la película elaborada con esta formulación tuvo un valor intermedio $\left(7,43 \times 10^{-4} \mathrm{~g}-\mathrm{mm} / \mathrm{Pa}-\mathrm{h}-\mathrm{m}^{2}\right)$ respecto a las demás formulaciones. Las películas con la mayor y la menor permeabilidad mostraron las tasas de respiración más altas.

Palabras clave: Recubrimiento, película comestible, biodegradable, permeabilidad, respiración.
\end{abstract}

\begin{abstract}
The coatings and films prepared whey (WPI) and chitosan (CHI) are known to be antifungal and also a barrier to the transference of water vapor, having an effect on the preservation of fruits. The aim of this study was to determine the effect of a coating of WPI and CHI on respiration of banana during storage at ambient conditions $\left(25 \pm 3{ }^{\circ} \mathrm{C}\right.$ and $75 \pm 5 \% \mathrm{RH}$ ) and its relation to water vapor permeability (PVA) of the films. Different concentrations of WPI and $\mathrm{CHI}$ were evaluated. $\mathrm{CHI}$ was used at $75 \%$ of deacetylation (solution of $1,2 \%, 1,5 \%$ and $1,8 \% \mathrm{w} / \mathrm{v}$ ) and WPI of $96 \%$ (solution $3 \%$ and $4 \% \mathrm{w} / \mathrm{v}$ ). The respiration rate was determined using a digital respirometer by measuring each sample during 55 minutes. The best coating was 1,5\% -WPI CHI 3\%. In this treatment the maturity and respiration was controlled and the climacteric peak occurred on day 9 of storage with $109,84 \mathrm{mg} \mathrm{CO} / \mathrm{kg}$-h (in the control sample occurred on day 7 with $172,71 \mathrm{mg} \mathrm{CO} / \mathrm{kg}-\mathrm{h}$ ). The water vapor permeability of the film made with this formulation had an intermediate value $\left(7,43 \times 10^{-4} \mathrm{~g}-\mathrm{mm} / \mathrm{m}^{2}-\mathrm{h}-\mathrm{Pa}\right)$ over other formulations. Films with the highest and the lowest permeability shown the highest respiration rates.

Keywords: Coating, edible film, biodegradable, permeability, respiration.
\end{abstract}

\section{Introducción}

El banano es una de las frutas más producidas y consumidas en el mundo debido a sus características distintivas de aroma y sabor (Gol y Ramana-Rao, 2011; Wall, 2006). Además, es una fuente barata de carbohidratos, rica en potasio, calcio, antioxidantes y otros micronutrientes. Por ser rico en azúcares y bajo en grasa, tiene diversos aprovechamientos en la industria alimentaria como alimento infantil, alimentos funcionales y postres (Mohapatra, et al., 2010). Sin embargo, esta fruta es altamente perecedera, debido a su alto contenido de agua, y es susceptible a muchas enfermedades, especialmente al ataque 
del hongo Colletotrichum musae (Bower, et al., 1998; Gol y Ramana-Rao, 2011; Bonilla, et al., 2012). Por ser un fruto climatérico, produce suficiente etileno para inducir rápidos cambios fisicoquímicos en propiedades tales como color, textura, aroma, composición química y frecuencia respiratoria (Bonilla, et al., 2012), la degradación de la clorofila y la reubicación de los micro y macronutrientes entre la pulpa y otras partes de la planta (Mohapatra, et al., 2010). Dentro de las actuales técnicas poscosecha del banano, se incluyen el uso de agentes de biocontrol, fungicidas, irradiación y otros tratamientos físicos, sustancias antimicrobianas naturales y compuestos orgánicos e inorgánicos (Ranasinghe, et al., 2002). Las investigaciones realizadas por Maqbool et al. (2010) y Win et al. (2007) aplicando recubrimientos en la conservación poscosecha de banano han logrado buenos resultados, entre ellos la maduración controlada del banano, la reducción del crecimiento del hongo y la extensión de la vida útil del banano en refrigeración hasta 28 días, lo que indica que es una tecnología promisoria que puede aportar beneficios en el gremio bananero.

El uso de recubrimientos en alimentos es una de las formas más rentables de incrementar su vida útil durante el almacenamiento (Beverly, et al., 2008). Uno de los componentes que más se han empleado en recubrimientos es la proteína aislada de lactosuero (WPI), la cual se distingue por su facilidad de formar recubrimientos con propiedad de barrera para la transferencia de vapor de agua y de gases (Prommakool, et al., 2011; Osés, et al., 2009). Por su parte, el quitosano (CHI) es un compuesto reconocido por tener propiedades antifúngicas $\mathrm{y}$ antimicrobiales, aunque con alta permeabilidad al vapor de agua (Miller y Krochta, 1997; Hernández, et al., 2011; Kurek, et al., 2014; Trejo, et al., 2001). Algunos recubrimientos y películas elaborados a partir de dichos compuestos han sido empleados en fresa (Park, et al., 2005), calabaza (Ponce, et al., 2008) y banano (Maqbool, et al., 2010). El objetivo de este trabajo fue determinar el efecto de un recubrimiento de WPI y CHI sobre la respiración en banano durante el almacenamiento y su relación con la permeabilidad al vapor de agua (PVA) de las películas.

\section{Materiales y Métodos}

Se realizó un diseño factorial aleatorizado con dos factores: concentración de WPI ( 2 niveles: $3 \%$ y $4 \%$ p/v) y concentración de CHI (3 niveles: 1,2, 1,5 y 1,8\% p/v). Los niveles empleados y el tiempo de estudio se definieron de acuerdo a ensayos preliminares. Las variables de respuesta fueron la respiración de los bananos recubiertos y la permeabilidad al vapor de agua de las películas elaboradas. Se evaluó la respiración diariamente durante 14 días. Para cada prueba se emplearon cuatro bananos garantizando suficiente $\mathrm{CO}_{2}$ en el respirómetro. Cada tratamiento se hizo por triplicado. Para la prueba de permeabilidad se elaboraron tres películas para cada tratamiento. Se realizó análisis de varianza y comparación de medias de Tukey con el programa estadístico SAS 9.2, con una confiabilidad del $95 \%$.

\section{Elaboración de la mezcla quitosano (CHI) - proteína de suero lácteo (WPI)}

Se disolvió el WPI (Davisco food, USA: Instantized BiPRO $96 \%)$ según el diseño experimental ( 3 y $4 \%$ p/v) en $100 \mathrm{ml}$ de agua destilada en un baño maría a $80{ }^{\circ} \mathrm{C}$ con agitación manual durante 20 minutos. Posteriormente se enfrió con agua a temperatura ambiente y se homogenizó en un equipo Ultraturrax durante $5 \mathrm{~min}$ a $9000 \mathrm{rpm}$.

Para la preparación de CHI (Sigma-Adrich, USA: $\geq 75 \%$ desacetilado) se hizo la disolución del quitosano en las concentraciones de interés $(1,2,1,5$ y 1,8\% p/v) en ácido acético glacial al $1 \%$ y agitación manual durante $40 \mathrm{~min}$ en temperatura ambiente $\left(25 \pm 2{ }^{\circ} \mathrm{C}\right)$. Posteriormente se realizó la homogenización de la solución utilizando el Ultraturrax a $9000 \mathrm{rpm}$ durante $5 \mathrm{~min}$.

Para realizar la mezcla, se adicionó la solución de WPI a la de CHI y se homogenizó empleando el Ultraturrax a 10000 rpm durante 25 min evitando la formación de espuma.

\section{Selección, recubrimiento y secado de las muestras}

Se seleccionaron bananos Gros Michel en escala 1 de coloración establecida por Piña et al. (2006). Se escogieron muestras de tamaño similar sin señales de ataques microbiológicos o de golpes. Se sumergió cada banano (incluyendo las puntas) durante 1 min en la solución. Para el secado, se suspendieron los bananos en una estructura metálica de tal manera que no tuvieran contacto con superficies durante $90 \mathrm{~min}$. Finalmente, se almacenaron a temperatura ambiental promedio $25 \pm 2{ }^{\circ} \mathrm{C}$ y humedad relativa de $75 \pm 2 \%$.

\section{Medición de la respiración}

Se evaluó la tasa de respiración ( $\mathrm{mg} \mathrm{CO} / \mathrm{kg}$-h) durante 14 días a $25 \pm 3{ }^{\circ} \mathrm{C}-70 \pm 5 \% \mathrm{HR}$ en bananos con y sin recubrimiento. Para determinar la tasa de respiración se estimó la cantidad de $\mathrm{CO}_{2}$ producida durante $55 \mathrm{~min}$ para cada tratamiento en un respirómetro digital construido en la Universidad del Valle (descrito en detalle por Ortega, et al., 2014). Se registró el peso de cada muestra, el tiempo de medición, el volumen del equipo, la densidad del producto y el promedio de los incrementos de los niveles de $\mathrm{CO}_{2}$. Para determinar la tasa respiratoria (mg de $\mathrm{CO}_{2} \mathrm{~kg}^{-1}$ hora $^{-1}$ ) se empleó la siguiente ecuación:

$$
T R=\frac{(1.8)(I C)(V R)}{(w)(I L)}
$$

donde, $T R$ es la tasa de respiración (mg kg-1h-1 $)$; IC es el incremento en la concentración de $\mathrm{CO}_{2}(\mathrm{mg}) ; V R$ es el volumen libre en el recipiente $\left(\mathrm{m}^{3}\right) ; w$ es el peso de la fruta $(\mathrm{kg}), I L$ es el intervalo de tiempo de lectura $(\mathrm{h})$ y el valor 1,8 corresponde a un factor de conversión de gases $\left(1 \mathrm{ppm}=1,8 \mathrm{mgCO}_{2} / \mathrm{m}^{3}\right)$.

\section{Elaboración de las películas}

Se vertieron $7 \mathrm{ml}$ de la solución CHI-WPI sobre una lámina lisa de polipropileno. La solución se esparció de tal manera que se alcanzara un diámetro de $12 \mathrm{~cm}$ y se dejó secar a temperatura ambiente $\left(25 \pm 3{ }^{\circ} \mathrm{C}\right.$ y $\left.75 \pm 2 \% \mathrm{HR}\right)$ durante dos días. El espesor de las películas fue de $0.049 \pm 0,0088 \mathrm{~mm}$, medido con un calibrador en tres puntos de cada película.

\section{Medición de permeabilidad de las películas al vapor de agua (PVA)}

La permeabilidad de las películas al vapor de agua (g-mm/ $\mathrm{Pa}-\mathrm{h}-\mathrm{m}^{2}$ ) se midió empleando el método de Debeaufort 


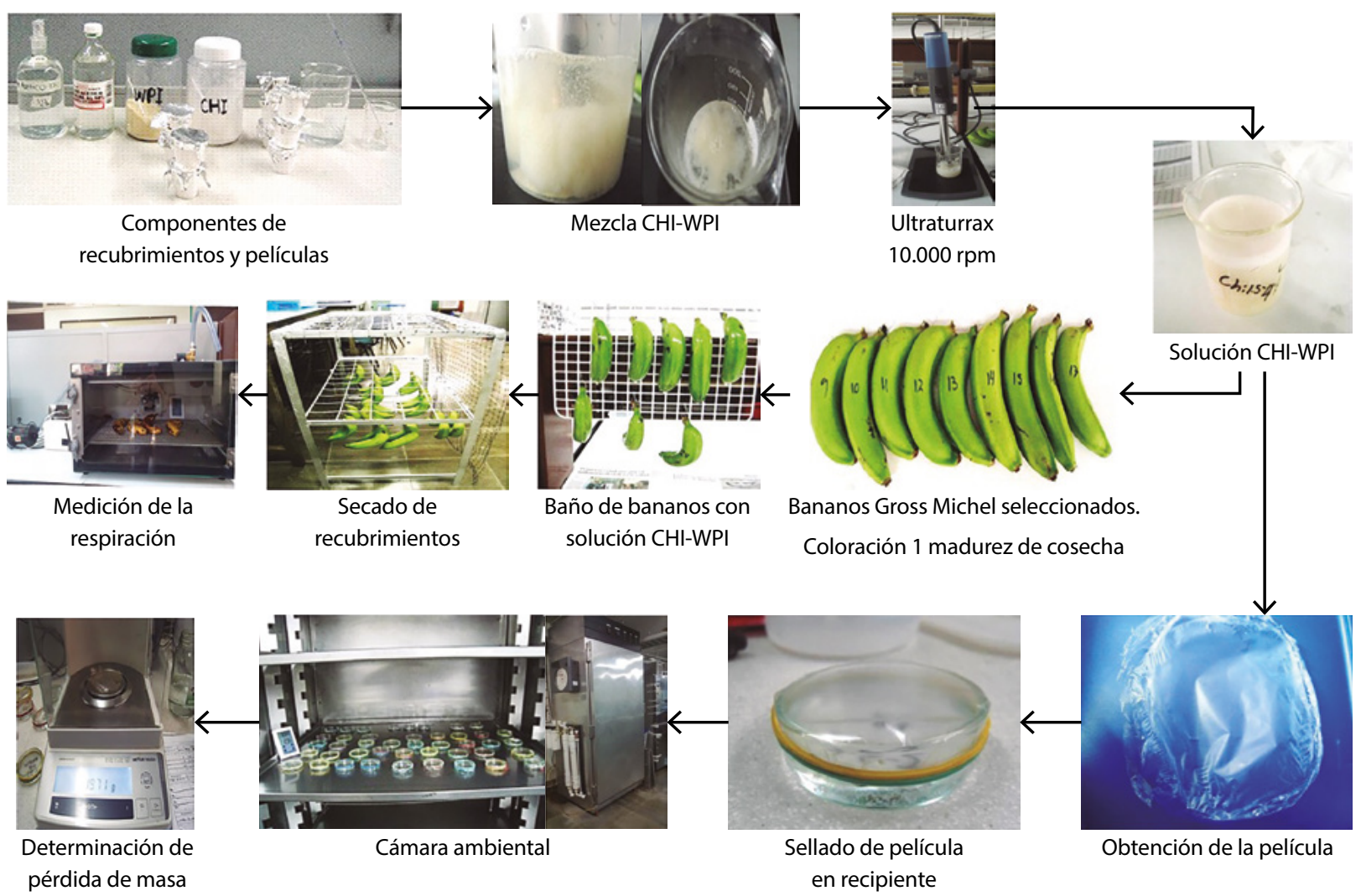

Figura 1. Metodología de formación de recubrimientos y películas de quitosano (CHI) y proteína de lactosuero (WPI).

et al. (1993). Se utilizaron cajas de petri de $4,95 \pm 1 \mathrm{~cm}$ y de $5,55 \pm 0,5 \mathrm{~cm}$ de diámetro. A cada caja de Petri se adicionaron $10 \mathrm{ml}$ de solución saturada de $\mathrm{MgCl}_{2} \mathrm{x}_{6} \mathrm{H}_{2} \mathrm{O}$ con $\mathrm{a}_{\mathrm{w}}$ ajustada a 0,33 y se cubrió la parte superior de la caja con la película, fijándola con una banda de caucho. Posteriormente se pesaron y se mantuvieron durante $24 \mathrm{~h}$ en una cámara ambiental (marca ADE Tecnología, Bogotá, Colombia) a $25 \pm 3{ }^{\circ} \mathrm{C}$ y $70 \pm 2 \%$ HR. Pasado este tiempo, se retiraron las muestras y se pesaron nuevamente. El área de transferencia de vapor de agua a través de la película $\left(\pi r^{2}\right)$ fue $1,97 \times 10^{-3} \pm 0,0001 \mathrm{~m}^{2}$ (caja pequeña) y $2,37 \times 10$ ${ }^{3} \pm 0,0001 \mathrm{~m}^{2}$ (caja grande). La PVA (g/m s Pa) se calculó de acuerdo a la siguiente ecuación:

$$
P V A=\frac{(\Delta m)(e)}{(\Delta t)(\Delta p)(A)}
$$

Donde: $\Delta m$ es la pérdida de peso (g); $e$ es el espesor de la película (m); $\Delta t$ es el tiempo de la prueba (s); $\Delta p$ es la diferencia de presiones parciales (calculadas como la humedad relativa del medio multiplicada por la presión de saturación a la temperatura del medio) entre los dos lados de la película $(\mathrm{Pa})$. Se supone una humedad relativa en el interior de la caja petri igual a cero y se tiene la humedad relativa en el exterior igual a $70 \%$ y la presión de saturación determinada a $25^{\circ} \mathrm{C}$ (condiciones de la cámara ambiental); $A$ es el área de la película expuesta a la transferencia de humedad $\left(\mathrm{m}^{2}\right)$. En la Figura 1 se presenta la metodología utilizada.

\section{Resultados y Discusión}

\section{Respiración de los bananos Gros Michel}

En la Figura 2 se presentan las curvas de respiración. Las muestras control presentaron el pico climatérico en el día 7, incrementando el valor de la tasa de respiración desde el día 1 hasta alcanzar un valor máximo $172,71 \pm 10 \mathrm{mg} \mathrm{CO} / \mathrm{kg} \mathrm{h}$. Este valor fue más alto que los picos observados en las muestras recubiertas. Según Khwaldia et al. (2004), la presencia de WPI en el recubrimiento proporciona menor intensidad en la respiración debido a que éste se encarga de regular la absorción de $\mathrm{O}_{2}$ y, por ende, la respiración. El pico climatérico entre los días 6 y 7 de almacenamiento es típico en bananos sin recubrimientos; este comportamiento ha sido reportado por otros autores como Elitzur et al. (2010), quienes almacenaron a $20^{\circ} \mathrm{C}$ y $75 \%$ HR durante 16 días, Kittur et al. (2001) a $27^{\circ} \mathrm{C}$, $65 \%$ HR y 14 días, y Biale et al. (1953) a $20^{\circ} \mathrm{C}$ durante 10 días.

En las muestras recubiertas, el pico climatérico varió entre el día 4 (tratamiento CHI 1,8\% - WPI 4\%), y el día 9 (tratamiento CHI 1,5\% - WPI 3\%). El mayor pico climatérico lo tuvieron las muestras con el recubrimiento $\mathrm{CHI} 1,2 \%$-WPI $4 \%$, las cuales mostraron un rápido incremento en su tasa respiratoria desde el día 1 hasta alcanzar el pico climatérico en el día 5 con $161 \pm 3 \mathrm{mgCO}_{2} / \mathrm{kg}$ h. Por su parte, las muestras con el recubrimiento $\mathrm{CHI} 1,8 \%$-WPI $4 \%$ fueron las que presentaron el pico climatérico más rápidamente (en el día 4). Durante todo el tiempo de estudio, las tasas de respiración más bajas con relación al control se observaron en los 
tratamientos CHI 1,2\%-WPI 3\%, CHI 1,5\%-WPI 3\% y CHI 1,5\%-WPI 4\%. Las muestras con recubrimiento CHI 1,5\%WPI 3\% tuvieron el pico climatérico con menor intensidad, el cual ocurrió en el mayor tiempo de almacenamiento.

Campuzano et al. (2010) y Barrero y Urrea (2012), quienes han investigado la variedad Gros Michel y los efectos logrados por acción de los recubrimientos, denominan este efecto como de atmósfera modificada debido a que se regula el intercambio de gases. Ese efecto de barrera puede causar menor deterioro como lo manifiesta Castellanos et al. (2011). Al comparar el número de días para llegar al pico climatérico, se observa que, en general, en los tratamientos con menor cantidad de WPI en la formulación el pico climatérico fue cercano o posterior al de la muestra control (6-9 días), mientras que en los tratamientos con mayor cantidad de WPI, tuvieron picos climatéricos entre 4 y 6 días.

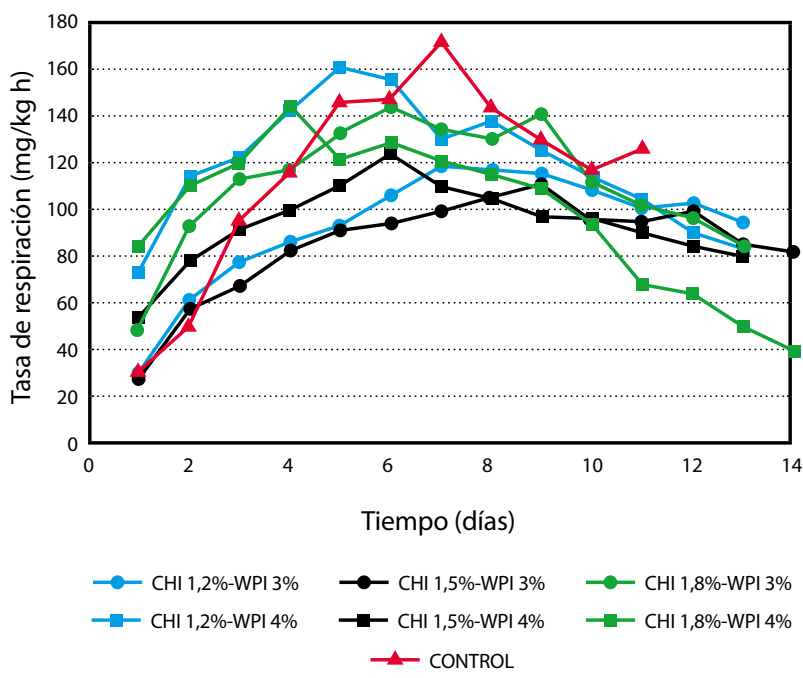

Figura 2. Efecto del recubrimiento de quitosano $(\mathrm{CHI})$ y proteína de lactosuero (WPI) sobre la respiración de bananos $\left(25 \pm 3{ }^{\circ} \mathrm{C}\right.$ y $\left.75 \pm 5 \% \mathrm{HR}\right)$

\section{Permeabilidad al vapor de agua (PVA)}

Los resultados observados en la tasa respiratoria están posiblemente influenciados por la permeabilidad de los recubrimientos al vapor de agua y por la acción sinérgica de los componentes del recubrimiento (WPI y CHI). En la Figura 3 se presenta la permeabilidad al vapor de agua de las películas elaboradas con igual formulación que los recubrimientos.

Los valores observados se encuentran dentro de los parámetros de permeabilidad al vapor de agua reportados por otros autores para componentes similares. Embuscado y Huber (2009) determinaron la permeabilidad de un recubrimiento de $\mathrm{CHI}$ $1 \%$-Almidón de maíz 5\%, con un valor de $0,5 \mathrm{E}-10 \mathrm{~g} / \mathrm{m} \mathrm{s} \mathrm{Pa}$; Lin y Zhao (2007) publicaron datos de permeabilidad de quitosano a $25^{\circ} \mathrm{C}$ y $50-70 \% \mathrm{HR}$ con un valor de $4,9 \mathrm{E}-10 \mathrm{~g} / \mathrm{m} \mathrm{s} \mathrm{Pa}$, y García et al. (2009) presentaron datos de permeabilidad de películas de quitosano $100 \%$ y en combinación con almidón de maíz al $5 \%$ con valores de $5,04 \mathrm{E}-10 \mathrm{~g} / \mathrm{m}$ s Pa y $8,76 \mathrm{E}-10$ $\mathrm{g} / \mathrm{m} \mathrm{s} \mathrm{Pa}$, respectivamente. Por su parte, Fakhreddin-Hosseini et al. (2013) presentaron datos de permeabilidad de películas de quitosano y gelatina de pescado a $25^{\circ} \mathrm{C}$ y $50 \% \mathrm{HR}$ de $0,6 \pm 0,034$ g mm/kPa h m² (1,67E-10 g/m s Pa) y Prommakool et al. (2011) reportaron valores de permeabilidad de proteína de lactosuero con $25 \%$ de glicerol de $2,6 \mathrm{~g} \mathrm{~mm} / \mathrm{kPa} \mathrm{h} \mathrm{m}^{2}(7,22 \mathrm{E}-10 \mathrm{~g} / \mathrm{m} \mathrm{s} \mathrm{Pa})$ a $23^{\circ} \mathrm{C}$ y $0 \% \mathrm{HR}$ (en este trabajo los valores de PVA están entre $1,49 \mathrm{E}-10$ y $2,50 \mathrm{E}-10 \mathrm{~g} / \mathrm{m} \mathrm{s} \mathrm{Pa}$ a $25^{\circ} \mathrm{C}$ y $70 \% \mathrm{HR}$ ambiente). $\mathrm{Al}$ observar estos resultados, se puede concluir que el CHI tiene mayor permeabilidad al vapor de agua que la WPI, por lo cual es usual combinar estos ingredientes para lograr el efecto antifúngico del CHI y reducir la permeabilidad al vapor de agua debido al WPI.

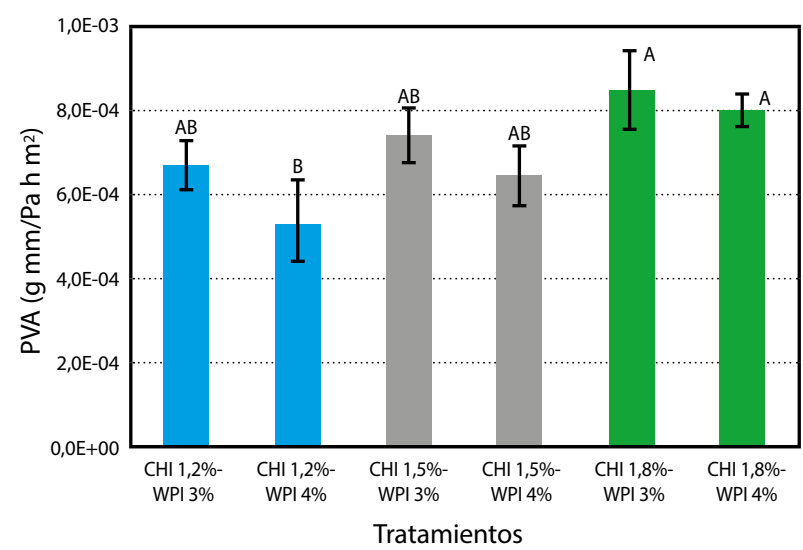

Figura 3. Permeabilidad al vapor de agua (PVA) de las películas de quitosano (CHI) y proteína de lactosuero (WPI).

Según la Figura 3 y la prueba de comparación de medias de Tukey $(p<0,05)$ en el modelo factorial, se encontraron tres grupos $(\mathrm{B}, \mathrm{AB}$ y $\mathrm{A})$. El primer grupo corresponde al tratamiento con la permeabilidad más baja (CHI 1,2\% - WPI 4\%), el segundo grupo incluye los tratamientos con permeabilidad media (CHI 1,5\% - WPI 4\%, CHI 1,2\% - WPI 3\%, CHI 1,5\% WPI 3\%) y el tercer grupo corresponde a los tratamientos con alta permeabilidad (CHI 1,8\% - WPI 3\%, CHI 1,8\% - WPI 4\%). Cuando se comparan estos grupos con los valores de tasa de respiración en el pico climatérico se observa que existe correlación entre ellos. Para el tratamiento del primer grupo se obtuvo una tasa de respiración de $161 \mathrm{mg} \mathrm{CO} / \mathrm{kg} \mathrm{h}$; para los tratamientos del segundo grupo se obtuvieron tasas de respiración de 124, 119 y $110 \mathrm{mg} \mathrm{CO}_{2} / \mathrm{kg} \mathrm{h}$, respectivamente, y para el tercer grupo se obtuvo una tasa de respiración de $144 \mathrm{mg} \mathrm{CO}_{2} / \mathrm{kg} \mathrm{h}$ en ambos tratamientos. Puede verse que para recubrimientos con permeabilidad intermedia, la tasa de respiración disminuye, lo cual es un comportamiento deseado. Por el contrario, para permeabilidades bajas o altas la tasa de respiración aumenta.

En cuanto al número de días para llegar al pico climatérico, los tratamientos con PVA extremos (grupos B y A) presentaron picos climatéricos adelantados en comparación con el tratamiento control. En el primer caso se presentó una maduración acelerada con presencia de hongos (análisis visual), debida a la baja concentración de $\mathrm{CHI}$, el cual tiene propiedades antifúngicas y alto contenido de agua (baja PVA), acompañada de una posible fermentación. En el segundo caso aparentemente se presentó una interrupción del proceso fisiológico evidenciado en la apariencia externa verde y en la textura blanda.

Por otra parte, de acuerdo al ANOVA se observó que los dos factores tuvieron efecto estadísticamente significativo $(\mathrm{p}<0.05)$ sobre la permeabilidad. La prueba de comparación de medias de Tukey en el modelo factorial mostró que el nivel 
3 de WPI es estadísticamente superior al nivel 4 de WPI, lo que significa que, en general, con mayor concentración de WPI se obtiene menor permeabilidad. En cuanto al CHI, se presentó diferencia estadística entre los niveles de CHI 1,8, 1,5 y 1,2, siendo la concentración CHI 1,8 la de mayor permeabilidad en las dos concentraciones de WPI.

El tratamiento CHI 1,5\% - WPI 3\% tuvo el mejor comportamiento, mostró un pico climatérico tardío respecto al control y un proceso de senescencia más suave, alcanzando los 14 días de prueba en buen estado (Figura 2). Este comportamiento se debe posiblemente a una sinergia CHI-WPI en la cual una permeabilidad intermedia provoca baja tasa de respiración y retardo del pico climatérico, con efecto antifúngico suficiente del CHI y con permeabilidad controlada por la presencia del WPI.

\section{Conclusiones}

Se observó un efecto significativo de CHI y WPI sobre la permeabilidad de las películas al vapor de agua; a mayor concentración de CHI y menor concentración de WPI se incrementó la permeabilidad. Adicionalmente, los tratamientos se comportaron de manera diferente a nivel estadístico, conformando tres grupos: los grupos con más baja permeabilidad y con más alta permeabilidad coincidieron con los grupos con mayor y menor tasa de respiración, respectivamente. Se concluye que es necesario tener permeabilidad intermedia para lograr un balance en la cantidad de humedad que se transfiere a través del recubrimiento, logrando de esta forma un pico climatérico tardío y una curva de respiración con menores variaciones. El mejor recubrimiento fue $\mathrm{CHI}$ 1,5\% - WPI 3\%, el cual permitió que los bananos realizaran la maduración y la respiración de manera controlada. El pico climatérico para este tratamiento ocurrió en el día 9 de almacenamiento, con una tasa de respiración de 109,84 mg CO $/ \mathrm{kg}$-h (en la muestra control ocurrió en el día 7, con una tasa de respiración de $172,71 \mathrm{mg} \mathrm{CO} / / \mathrm{kg}$-h). La permeabilidad al vapor de agua de la película elaborada con esta formulación tuvo un valor intermedio $\left(7,4 \times 10^{-4} \mathrm{~g}-\mathrm{mm} /\right.$ $\mathrm{Pa}-\mathrm{h}-\mathrm{m}^{2}$ ) respecto a las demás formulaciones, con lo que se logra un equilibrio en la transferencia de vapor de agua.

Se recomienda para futuros trabajos evaluar la permeabilidad al dióxido de carbono y al oxígeno. La transferencia de estos gases tiene un efecto importante en el proceso de respiración y la maduración.

\section{Referencias}

Barrero, M. O. y Urrea, J. W., 2012. Modelamiento de la tasa de respiración de banano, variedad Gross Michel, basado en las ecuaciones de la cinética enzimática de MichaelisMenten. En: Tumbaga, 7, pp.101-114.

Beverly, R., Janes, M. E., Prinyawiwatkul, W., No, H. K. y Beverly, R. L., 2008. Edible chitosan films on ready-to-eat roast beef for the control of Listeria monocytogenes. En: Food Microbiology, 25(3), pp.534-537.

Biale, J., Young, R. y Olmstead, A., 1953. Fruit respiration and ethylene. En: Plan Physiology, 29(2), pp.168-174.

Bonilla, J., Atarés, L., Vargas, M. y Chiralt, A., 2012. Edible films and coatings to prevent the detrimental effect of oxygen on food quality: Possibilities and limitations. En: Journal of Food Engineering, 110(2), pp.208-213.
Bower, J., Jobling, J., Patterson, B. y Ryan, D., 1998. A method for measuring the respiration rate and respiratory quotient of detached plant tissues. En: Postharvest Biology and Technology, 13(3), pp.263-270.

Castellanos, D. A., Algecira, N. A. y Villota, C. P., 2011. Aspectos relevantes en el almacenamiento de banano en empaques con atmósferas modificadas. En: Revista Iberoamericana de Tecnología de Postcosecha, 12(2), pp.114-134.

Campuzano, A., Cornejo, F., Ruiz, O. y Peralta, E., 2010. Efecto del Tipo de Producción de Banano Cavendish en su Comportamiento Poscosecha Resumen. En: Revista Tecnológica ESPOL-RTE, 23(2), pp.41-48.

Debeaufort, F., Martin-Polo, M. y Voilley, A., 1993. Polarity homogeneity and structure affect water vapor permeability of model edible films. En: Journal of Food Science, 58(2), pp.426-429.

Elitzur, T., Vrebalov, J., Giovannoni, J., Goldschmidt, E. y Haya, F., 2010. The regulation of MADS-box gene expression during ripening of banana and their regulatory interaction with ethylene. En: Journal of Experimental Botany, 61(5), pp.1523-35.

Embuscado M. y Huber K., 2009. Edible films and coatings for food applications. New York: Springer-Verlag, p.403.

Fakhreddin-Hosseini, S., Rezaei, M., Zandi, M. y Ghavi, F.F., 2013. Preparation and functional properties of fish gelatinchitosan blend edible films. En: Food Chemistry, 136(3-4), pp.1490-1495.

García, M. A., Pinotti, A., Martino, M. N. y Zaritzky, N. E., 2009. Characterization of starch and composite edible films and coatings. En: Embuscado, M., Huber, K., 2009. Edible films and coatings for food applications. New York: Springer-Verlag, pp.169-210.

Gol, N. B. y Ramana Rao, T. V., 2011. Banana fruit ripening as influenced by edible coatings. En: International Journal of Fruit Science, 11(2), pp.119-135.

Hernández, O. L., González, G., Gutierrez, M. N., Muñoz C, L.N. y Quintero R. A., 2011. Estudio de la actividad antibacteriana de películas elaboradas con quitosano a diferentes pesos moleculares incorporando aceites esenciales y extractos de especias como agentes antimicrobianos. En: Revista Mexicana de Ingeniería Química, 10(3), pp.455-463.

Khwaldia, K., Perez, C., Banon, S., Desobry, S. y Hardy, J., 2004. Milk proteins for edible films and coatings. En: Critical Reviews in Food Sci. and Nutrition, 44(4), pp.239-251.

Kittur, F.S, Saroja, N., Habibunnisa y Tharanathan, R. N., 2001. Polysaccharide-based composite coating formulations for shelf-life extension of fresh banana and mango. En: European Food Research and Technology, 213(4-5), pp.306-311.

Kurek, M., Guinault, A., Voilley, A., Galic, K. y Debeaufort, F., 2014. Effect of relative humidity on carvacrol release and permeation properties of chitosan based films and coatings. En: Food Chemistry, pp.144, 9-17.

Lin, D. y Zhao, Y., 2007. Innovations in the development and application of edible coatings for fresh and minimally processed fruits and vegetables. En: Comprehensive Reviews in Food Science and Food Safety, 6(3), pp.60-75.

Maqbool, M., Ramachandran, S., Smith, D.R., Alderson, P., Ali y A., 2010. Control of postharvest anthracnose of banana using a new edible composite coating. En: Crop Protection, 29 (10), pp.1136-1141. 
Miller, K. S. y Krochta, J. M., 1997. Oxygen and aroma barrier properties of edible films: a review. En: Trends in Food Science and Technology, 8(7), pp.228-237.

Mohapatra, D., Mishra, S. y Sutar, N., 2010. Banana post harvest practices: Current status and future prospectsA review. En: Agricultural Reviews, 31(1), pp.56-62.

Ortega, K.J., Gómez, C.A., Vélez, C., Velasco, J. y Alonso, L. (2014). Desarrollo de un respirómetro para productos agrícolas. En: Revista de la Facultad Nacional de Agronomía Medellín, 67(2),pp.1116-1119.

Osés, J., Fabregat-Vázquez, M., Pedroza-Islas, R., Tomás, S. A., Cruz-Orea, A. y Maté, J. I., 2009. Development and characterization of composite edible films based on whey protein isolate and mesquite gum. En: Journal of Food Engineering, 92(1), pp.56-62.

Park, S.I., Stan, S.D., Daeschel, M.A., Zhao, Y.Y., 2005. Antifungal Coatings on Fresh Strawberries (Fragaria $\times$ ananassa) to Control Mold Growth During Cold Storage. En: Food Microbiology and Safety, 70(4), pp.202-207.

Piña, G., Laborem, G., Surga, J., Marín, C., Rangel, L., Espinoza M., Delgado, A., 2006. Atributos de calidad en frutos de híbridos FHIA (Musa) para tres ciclos de cosecha. En: Revista Facultad de Agronomía de La Universidad de Zulia (LUZ), 23(1), pp.425-442.

Ponce, A.G., Roura, S.I., del Valle, C.E. y Moreira, M.R., 2008. Antimicrobial and antioxidant activities of edible coatings enriched with natural plant extracts: In vitro and in vivo studies. En: Postharvest Biology and Technology, 49(2), pp.294-300.

Prommakool, A., Sajjaanantakul, T., Janjarasskul, T. y Krochta, J. M., 2011. Whey protein - okra polysaccharide fraction blend edible films: tensile properties, water vapor permeability and oxygen permeability. En: Journal of the Science of Food and Agriculture, 91(2), pp.362-369.

Ranasinghe, L., Jayawardena, B. y Abeywickrama, K., 2002. Fungicidal activity of essential oils of Cinnamomum zeylanicum (L.) and Syzygium aromaticum (L.) Merr et L.M. Perry against crown rot and anthracnose pathogens isolated from banana. En: Letters in Applied Microbiology, 35(3), pp.208-11.

Trejo, V., Aragon, N. y Miranda, P., 2001. Estimación de la permeabilidad al vapor de agua en películas a base de quitosán. En: Revista de la Sociedad Química de México, 45(1), pp.1-5.

Wall, M. M., 2006. Ascorbic acid, vitamin A, and mineral composition of banana (Musa sp.) and papaya (Carica papaya) cultivars grown in Hawaii. En: Journal of Food Composition and Analysis, 19(5), pp.434-445.

Win, N. K. K., Jitareerat, P., Kanlayanarat, S. y Sangchote, S., 2007. Effects of cinnamon extract, chitosan coating, hot water treatment and their combinations on crown rot disease and quality of banana fruit. En: Postharvest Biology and Technology, 45(3), pp.333-340. 